\title{
Quality of Life After Definitive Linear Accelerator- Based Stereotactic Radiotherapy for Prostate Cancer: A Longitudinal Study
}

Hideomi Yamashita ( $\nabla$ yamashitah-rad@h.u-tokyo.ac.jp )

The University of Tokyo Hospital

Mami Ogita

The University of Tokyo Hospital

Subaru Sawayanagi

The University of Tokyo Hospital

\section{Yuki Nozawa}

The University of Tokyo Hospital

Osamu Abe

The University of Tokyo Hospital

\section{Research Article}

Keywords: Stereotactic body radiotherapy, prostate cancer, quality of life, patient-reported outcomes, radiation therapy

Posted Date: March 9th, 2022

DOI: https://doi.org/10.21203/rs.3.rs-1036625/v2

License: (c) (i) This work is licensed under a Creative Commons Attribution 4.0 International License. Read Full License 


\section{Abstract \\ Background}

Prostate cancer is the second most common malignancy worldwide, and the majority of patients are diagnosed with localized disease. We examined patients' quality of life after stereotactic body radiation therapy (SBRT) for prostate cancer.

\section{Method}

We included patients who were treated between 2016 and 2020. Inclusion criteria were adenocarcinoma of the prostate; class risk of low, intermediate, and high; and a World Health Organization performance status of 0-2. Quality of life was measured using the Functional Assessment of Cancer Therapy-Prostate (FACT-P).

\section{Results}

A total of 439 patients were treated with SBRT, with a median age of 73 years old. The median follow-up period was 34 months. FACT-P Trial Outcome Index ( $p<0.0001)$, FACT-General $(p=0.0003)$, and FACT-PTotal $(p<0.0001)$ scores declined at 1 month post-SBRT, then recovered and returned to the same level as before treatment at 3-4 months post-SBRT. The decrease in quality of life in the first month was particularly remarkable in patients who received long-term hormone injections (36\%). One month after the end of SBRT, about $22 \%$ of patients experienced "quite a bit" or more troubling side effects.

\section{Conclusions}

This study showed longitudinal changes in quality of life by FACT-P after SBRT for prostate cancer. Overall, prostate SBRT was well tolerated.

\section{Introduction}

Prostate cancer (PC) is the second most common malignancy worldwide, and the majority of PC patients are diagnosed with localized disease (1). There are multiple efficacious guideline-recommended treatment options for localized PC, including radical prostatectomy, external beam radiation, and brachytherapy. Recent American Society for Radiation Oncology/American Society of Clinical Oncology/American Urological Association guidelines (2) included recommendations regarding the use of stereotactic body radiotherapy (SBRT) for PC. According to the National Comprehensive Cancer Network (NCCN) Guidelines Version 1.2021 (3), five fractions of 7.25-8 Gy radical SBRT for PC are indicated for low-risk to high- and ultra-high-risk cases. There is increasing awareness that quality of life (QoL) alongside objective measures of late adverse genitourinary (GU) and gastrointestinal (GI) toxicity 
are essential for the decision-making process in the management of patients with PC. To the best of our knowledge, there have been no reports evaluating QoL fluctuations after radical prostate SBRT using the Functional Assessment of Cancer Therapy-Prostate (FACT-P) questionnaire. Therefore, the present study investigated the long-term QoL of patients after SBRT using a valid and self-administered QoL questionnaire via the FACT-P.

\section{Materials And Methods}

We conducted a prospective cohort study of patients with PC treated with SBRT at our institution (University of Tokyo Hospital). The inclusion criteria were as follows: (1) histologically proven adenocarcinoma of the prostate; (2) NCCN class risk of low, intermediate, or high; and (3) World Health Organization (WHO) performance status of $0-2$. The exclusion criteria were as follows: (1) lymph node metastasis $(n=12),(2)$ bone metastasis $(n=11),(3)$ castration-resistant PC $(n=7),(4)$ after radical prostatectomy $(n=1)$, (5) SBRT for local recurrence after external beam radiation therapy $(n=2),(6)$ local recurrence after high-intensity focused ultrasound (HIFU) $(n=3)$, and (7) receiving more or less than five fractions of radiotherapy $(n=1)$. We did not limit the prostate volume or baseline International Prostate Symptom Score to maximize the number of patients included.

\section{Combination hormone therapy}

Hormone therapy was administered over a short term of 4-6 months in the intermediate -risk group, and over a longer term of 1.5-2 years in the high-risk group. There was no concomitant use of bicalutamide, the first Degarelix injection, and the second and subsequent leuprorelin injections. SBRT was performed when prostate-specific antigen (PSA) dropped to near $1.0 \mathrm{mg} / \mathrm{mL}$. However, SBRT was started by the second month in the short-term administration group and by half a year in the long-term administration group.

\section{SBRT method}

The clinical target volume (CTV) included the prostate and the proximal $2 \mathrm{~cm}$ of the seminal vesicle (SV) for high-risk patients, the prostate and $1 \mathrm{~cm}$ of the SV for intermediate-risk patients and the prostate only for low-risk patients. The planning target volume (PTV) consisted of a CTV with a posterior $3 \mathrm{~mm}$ margin and a $5 \mathrm{~mm}$ margin for all other margins.

The irradiation dose was PTV 95\% prescription. 36.25 Gy in five fractions was $54 \%$, and 40 Gy in five fractions was $43 \%$. The former had been adopted before July 2018 and the latter after that. SBRT was performed using volumetric modulated arc therapy (VMAT) with flattening filter-free beams using a linear accelerator with image-guidance. Image guidance was performed using daily cone-beam computed tomography (CBCT). SBRT was performed five times every other day, excluding weekends. There were many cases of 177 patients in which SpaceOAR hydrogel (SpaceOAR; Augmenix Inc.) was applied before SBRT. Our previous report (4) describes the spacer insertion method, magnetic resonance imaging (MRI) planning, computed tomography (CT) scan for SBRT, definition of the target volume, and the organs at 
risk. Each patient was given a glycerin enema of 30 or 40 cc 2 hours before the examination or SBRT. After that, the patients were asked to drink water and hold their urine for 2 hours. From 4 days before the examination to the last day of SBRT, the patients were encouraged to take elobixibat hydrate or polyethylene glycol daily. SBRT dose constraints are shown in Table 1.

Table 1. Dose constraint of $40 \mathrm{~Gy}$ in 5 fractions

\begin{tabular}{|c|c|c|c|c|c|}
\hline \multicolumn{2}{|c|}{ Dose constraint } & \multicolumn{2}{|c|}{ With SpaceOAR } & \multicolumn{2}{|c|}{ Without SpaceOAR } \\
\hline Prostate & Volume & Goal & Tolerance & Goal & Torelance \\
\hline Urethra & $\begin{array}{l}\text { Max } \\
\text { Max }\end{array}$ & & & & \\
\hline \multirow[t]{4}{*}{ PTV } & V110\% & $<0.5 \%$ & $<2 \%$ & $<0.5 \%$ & $<2 \%$ \\
\hline & D99\% & $>39.5 \mathrm{~Gy}$ & $>39.17$ Gy & $>39.2 \mathrm{~Gy}$ & $>38.1 \mathrm{~Gy}$ \\
\hline & Max & $<43.26 \mathrm{~Gy}$ & $<44.14$ Gy & $<43.9$ Gy & $<45.5 \mathrm{~Gy}$ \\
\hline & Mean & $<41.16$ Gy & $<41.71 \mathrm{~Gy}$ & $<41.5 \mathrm{~Gy}$ & $<42$ Gy \\
\hline \multirow[t]{4}{*}{ Bladder } & V40Gy & $<3.7 \%$ & $<6 \%$ & $<4.6 \%$ & $<6 \%$ \\
\hline & V20Gy & $<22.8 \%$ & $<35 \%$ & $<27.4 \%$ & $<49 \%$ \\
\hline & V40.83Gy & $<6.2 \mathrm{cc}$ & $<8 \mathrm{cc}$ & $<6.9 \mathrm{cc}$ & $<10 \mathrm{cc}$ \\
\hline & Max & $<42.7$ Gy & $<44.14$ Gy & $<43.4$ Gy & $<45$ Gy \\
\hline \multirow[t]{7}{*}{ Rectum } & Mean & $<11.92$ Gy & $\begin{array}{c}<16.55 \text { Gy } \\
\text { m above an }\end{array}$ & $\begin{array}{r}<13.7 \text { Gy } \\
\text { d below the }\end{array}$ & $\begin{array}{l}<20 \mathrm{~Gy} \\
\mathrm{TV}\end{array}$ \\
\hline & V40Gy & $<0.5 \%$ & $<1.5 \%$ & $<2.5 \%$ & $<4 \%$ \\
\hline & V36Gy & $<2.5 \%$ & $<5.7 \%$ & $<8 \%$ & $<11 \%$ \\
\hline & $\begin{array}{l}\text { V32Gy } \\
\text { V30Gy }\end{array}$ & $\begin{array}{l}<5 \% \\
<6.8 \%\end{array}$ & $\begin{array}{l}<10 \% \\
<13 \%\end{array}$ & $\begin{array}{l}<13 \% \\
<16 \%\end{array}$ & $\begin{array}{l}<17 \% \\
<20 \%\end{array}$ \\
\hline & V20Gy & $<28.2 \%$ & $<34 \%$ & $<32 \%$ & $<39 \%$ \\
\hline & $\operatorname{Max}$ & $<40.83 \mathrm{~Gy}$ & $<42.48$ Gy & $<42.7$ Gy & $<43.9$ Gy \\
\hline & Mean & $<16.88 \mathrm{~Gy}$ & $<18.21 \mathrm{~Gy}$ & $<17.4 \mathrm{~Gy}$ & $<19.6$ Gy \\
\hline Femoral head & $\underset{\text { Max }}{\operatorname{Max}}$ & $<17.66$ Gy & $<18.76$ Gy & $<17.66$ Gy & $<18.76 \mathrm{~Gy}$ \\
\hline Penile bulb & $\operatorname{Max}$ & $<40$ Gy & & $<40$ Gy & \\
\hline Small bowel & V30Gy & $0 \mathrm{cc}$ & $<5 c c$ & Occ & $<5 c c$ \\
\hline
\end{tabular}

\section{Description of the patient cohort}

Baseline patient characteristics are shown in Table 2. The median follow-up period was 34 months (maximum: 54 months). Between May 2016 and December 2020, 439 patients answered the FACT-QoL questionnaire. These 439 patients were all that had been treated with SBRT for PC with curative intent at our institution during this period. The median age of the sample was 73 years and the median PSA before all treatments was $9.2 \mathrm{ng} / \mathrm{mL}$. Clinical stage T2a was the most prevalent T-stage, with $47 \%$ of cases diagnosed as such. Regarding Gleason Score, $28 \%$ and $27 \%$ of patients were classified as Group 2 and 3 , 
respectively. Moreover, $53 \%$ of patients were classified as intermediate-risk, while $42 \%$ were classified as high-risk. Short-term hormones were given for 4-6 months to $42 \%$ of patients, while long-term hormones were given to $36 \%$ of patients. A total of 177 patients (40\%) had a hydrogel spacer inserted between their prostate and rectum before SBRT.

Table 2. Patient characteristics

\begin{tabular}{|c|c|c|}
\hline Factors & $\mathbf{N}$ & $\%$ \\
\hline Total & 439 & 100 \\
\hline \multicolumn{3}{|l|}{ Age (years old) } \\
\hline Median (range) & \multicolumn{2}{|c|}{$73(33-92)$} \\
\hline Quartile & \multicolumn{2}{|c|}{68 and 78} \\
\hline \multicolumn{3}{|l|}{ iPSA (ng/mL) } \\
\hline Median (range) & \multicolumn{2}{|c|}{$9.2(1.6-24.0)$} \\
\hline Quartile & \multicolumn{2}{|c|}{6.3 and 14.7} \\
\hline \multicolumn{3}{|l|}{ ClinicalT-stage } \\
\hline $\mathrm{T} 1 \mathrm{c}$ & 84 & 19.1 \\
\hline $\mathrm{T} 2 \mathrm{a}$ & 208 & 47.4 \\
\hline $\mathrm{T} 2 \mathrm{~b}$ & 20 & 4.6 \\
\hline $\mathrm{T} 2 \mathrm{c}$ & 61 & 13.9 \\
\hline T3a & 46 & 10.5 \\
\hline $\mathrm{T} 3 \mathrm{~b}$ & 14 & 3.2 \\
\hline $\mathrm{T} 4$ & 6 & 1.4 \\
\hline \multicolumn{3}{|l|}{ Gleason s core group } \\
\hline Group 1 & 46 & 10.5 \\
\hline Group 2 & 121 & 27.6 \\
\hline Group 3 & 117 & 26.7 \\
\hline Group 4 & 82 & 18.7 \\
\hline Group 5 & 73 & 16.6 \\
\hline \multicolumn{3}{|l|}{ Risk group } \\
\hline Low & 21 & 4.8 \\
\hline Intermediate-low & 84 & 19.1 \\
\hline Intermediate-high & 149 & 33.9 \\
\hline High & 112 & 25.5 \\
\hline Ver-high & 73 & 16.6 \\
\hline \multicolumn{3}{|l|}{ Hormonal therapy } \\
\hline None & 98 & 22.3 \\
\hline Short term & 183 & 41.7 \\
\hline Long term & 158 & 36.0 \\
\hline \multicolumn{3}{|l|}{$\mathrm{RT}$ total dose } \\
\hline $36.25 \mathrm{~Gy}$ & 239 & 54.4 \\
\hline $40 \mathrm{~Gy}$ & 187 & 42.6 \\
\hline $42.5 \mathrm{~Gy}$ & 13 & 3.0 \\
\hline
\end{tabular}

Abbreviation: PSA = prostate-specific antigen, RT = radiation therapy

\section{Follow-up}

Follow-up intervals were calculated from the date of the last SBRT dose. Outpatient follow-up was conducted at 1 month and 3-4 months after the end of SBRT, then at every 3 months until 2-year followup, and finally at every 6 months after the second year. We asked patients to complete a QoL 
questionnaire at each follow-up visit from May 2016. PSA was measured at every visit, and when it exceeded $2.0 \mathrm{ng} / \mathrm{mL}$ twice in a row, contrastenhanced MRI and/or 18F-fluorodeoxyglucose (FDG) positron emission tomography (PET) examination was added to check for recurrence, since prostate specific membrane antigen (PSMA) was not be approved at the moment in Japan. When treatment such as chemotherapy was started in the case of double cancers, it was excluded from the survey.

\section{Statistical analysis}

Risk classification was based on the NCCN risk classification v1. 2021 for PC. The paired t-test was used for comparison of the baseline. All p-values were two-sided, and $p<0.05$ was considered statistically significant. The FACT-P instrument is a multidimensional, self-administered, 39-item questionnaire. A FACT-P total score (FACT-P-Total) was obtained by adding together each patient's emotional well-being (EWB), family well-being (FWB), physical well-being (PWB), social well-being (SWB), and prostate cancer subscale (PCS) (range 0-152) scores. The FACT-General (FACT-G) total score by determined by adding together the EWB, FWB, PWB, and SWB (range 0-104) scores. The FACT-P Trial Outcome Index (TOI) was determined by adding the FWB, PWB, and PCS subscale scores (range $0-104$ ). Scores were obtained according to version 4 of the FACT scoring guidelines (5). Prorating was carried out only if more than $50 \%$ of the items on each subscale were answered. For all scales, the higher the score, the better the QoL. This study was approved by the Institutional Review Board of Tokyo University (No. 3372-6). All methods were performed in accordance with the relevant guidelines and regulations. Informed consent was obtained from all participants.

\section{Results}

The baseline prostate volume was $39.3 \mathrm{cc}$ (SD: 20.9) and the baseline international prostate symptom score was 9.9 (SD: 1.5).

Since we included all patients received SBRT in this survey, $28.5 \%$ of patients did not complete the questionnaire before treatment. In other words, although the questionnaire was distributed to all 439 patients, 314 patients $(71.5 \%)$ answered it before treatment and $242(55 \%)$ at one month. Even after that, the questionnaire continued to be distributed to all 439 patients every time they came to the outpatient department, everyone answered it at least once at some point. The number of the returned questionnaires at each point was shown in the bottom of Fig. 1. Across the FACT P-TOI, FACT-G, and FACT-P-Total, the QoL score at 1 month after the end of SBRT was significantly lower than that before SBRT (Fig. 1). The average QoL score dropped from 78.84 (standard error: 0.77) before SBRT to 72.35 (1.02) at 1 month in the FACT P-TOI ( $p<0.0001$ by t-test), from $79.91(0.79)$ to $75.38(0.96)$ in the FACT-G $(p=0.0003)$, and from $114.22(1.05)$ to $105.78(1.31)$ in the FACT-P-Total $(p<0.0001)$. An improving trend was then seen in the second month (average score: 74.62 [SE: 2.57], 77.13 [2.09], and 107.88 [3.23], respectively). Furthermore, at 3-4 months, the scores of the FACT P-TOI ( $p=0.0011$, average score: 76.80 [SE: 0.88 ]), FACT-G $(p=0.0410,78.15$ [0.94]), and FACT-P-Total $(p=0.0029,111.19$ [1.24]) improved significantly when compared with those recorded in the first month. There was no significant decrease in the QoL score during the subsequent follow-up period, until months 49-54. 
In the long-term hormone group, the QoL reduction seen in the FACT P-TOI (67.99) and FACT P-Total (102.08) at 1 month were larger than those in the short-term hormone group and the hormone-free group (Fig. 2). In the hormone-free group, QoL was lowest at two months instead of one month. With respect to use of a hydrogel spacer, the score of FACT P-TOI, FACT-G, and FACT P-Total at 1 month was 71.35 (SE: 1.02), 74.41 (0.96), and 104.26 (1.31) without spacer versus 74.97 (1.76) ( $p=0.11$ by unpaired $t$-test), $77.94(1.69)(p=0.10)$, and $109.74(2.28)(p=0.062)$ with spacer, respectively. With respect to the SBRT dose, the FACT P-TOI, FACT-G, and FACT-P-Total scores at 1 month were 72.58 (SE: 1.17), 75.22 (1.09), and 105.60 (1.46) after $36.25 \mathrm{~Gy}(\mathrm{p}=0.33$ of $36.25 \mathrm{~Gy}$ versus $40 \mathrm{~Gy}, \mathrm{p}=0.68$ of $36.25 \mathrm{~Gy}$ versus $42.5 \mathrm{~Gy}$, and $p=0.99$ of $40 \mathrm{~Gy}$ versus $42.5 \mathrm{~Gy})$, and $71.77(2.21), 76.05(2.16)$, and $106.39(3.02)(p=0.71,0.82$, and 0.74) after $40 \mathrm{~Gy}, 71.85(6.29), 74.15(5.47)$, and $105.15(7.88)(p=0.79,0.84$, and 0.88$)$ after $42.5 \mathrm{~Gy}$, respectively.

P7 (I have difficulty urinating) (average score: 3.25 before SBRT, 2.49 at 1 month, and 3.14 at 2 months) and BL2 (I urinate more frequently than usual) $(2.58,1.62$, and 2.22 , respectively) were the lowest 1 month after the end of SBRT (both p < 0.0001) (Fig. 3). Improvement was observed at 2 months and was fully recovered by months 3-4. P6 (I have trouble moving my bowels) and P8 (My problems with urinating limit my activities) continued to decline until the second month and recovered by months 3-4 (Fig. 3).

The percentage of those who answered that they were suffering from the side effects of GP5 (I am bothered by side effects of treatment) was also the highest in the first month after SBRT at $22 \%$. While this figure had increased from the $8 \%$ recorded during the pre-SBRT period, it then dropped to $11 \%$ by months 3-4 (Fig. 4). Subsequently, this percentage gradually decreased over time. By including those who were "somewhat," bothered by side effects, this result changed from $15 \%$ before SBRT to $45 \%$ at the 1 st month, $32 \%$ at the 2 nd month, and $28 \%$ at 3-4 months.

\section{Discussion}

In this prospective study, we evaluated the QoL of 439 patients treated with SBRT for low-, intermediate, and high-risk PC. To date, most QoL evaluations using FACT-P after SBRT for PC have only done so for metastatic cases (6-8). However, since these studies did not irradiate the primary prostate lesion, their results cannot be compared with the QoL after radical SBRT. Using the FACT-P questionnaire, it is highly novel to see the time-series changes in QoL after radical PC SBRT. The FACT-P has also been used in many phase III studies on PC (9-18). In this report, QoL declined at 1 month after the last SBRT, then recovered, and returned to the same level as before treatment by 3-4 months. The decrease in QoL in the first month was particularly remarkable in patients who received combined long-term hormone injections. One month after the last SBRT, about $22 \%$ of people were "quite a bit or very much" bothered by the side effects experienced. Since the QoL score of patient-reported side effects (i.e., P6, P7, P8, and BL2) also declined at the same time, this QoL change would be due to SBRT-induced acute toxicity, although we did not compare patient-reported outcome measures (PROMS) with toxicity assessment. In this study, we could not report data on the correlation between physician- and patient-reported outcomes. 
According to the previous reports (19-26), physician-reported acute GI and GU toxicity of grade 3-5 after SBRT of 2,4 , or 5 fractions were $0-2 \%$ and $0-2.5 \%$, respectively. In our study, $45 \%$ of patients answered "somewhat" or more to GP5 (I am bothered by side effects of treatment) at 1 month after the last SBRT. Moreover, $22 \%$ of patients responded that they were "quite a bit" or more troubled by the treatment's side effects. In general, patient-reported side effects are more frequent than physician-reported side effects.

According to the previous reports (19-26), physician-reported chronic GI and GU toxicity of grade 3-5 after SBRT were $0-7 \%$ and $0-5.5 \%$, respectively. Periprostatic hydrogel placement followed by prostate SBRT may result in minimal GI toxicity. In our study, $10-30 \%$ and $0-13 \%$ of patients answered "somewhat" or more and "quite a bit" or more in GP5 after 3-4 months, respectively, although these figures could not be compared directly since there was a difference of physician- and patient-reported outcome.

There were times when the response rate was small, such as after the 38th month. Since we included all patients treated with SBRT during this period, only $71 \%$ of patients had a baseline assessment and $55 \%$ were evaluated at 1 month. Thus, our QoL assessments may follow a heterogeneous distribution.

Previous studies have investigated the QoL change after conventional intensity-modulated radiation therapy (IMRT), but unlike our study, included an initial questionnaire 3 months after irradiation and found no decrease in QoL (27-29). In contrast, we collected novel QoL data before SBRT, as well as during the first and second months after SBRT. Moreover, we compared our findings with previous QoL data of patients treated using low dose rate (LDR) and high dose rate (HDR) brachytherapy. According to Slevin et al. (30), maximal deterioration in mean urinary $(p<0.001)$ and sexual summary scores using a validated Expanded Prostate Cancer Index Composite (EPIC) questionnaire was noted 6 weeks after implantation, with severe urinary symptoms and moderate bowel/sexual symptoms after HDR brachytherapy. At 6 months, urinary and bowel QoL improved to involve only mild impairment, which was fully resolved at 10 months (30). According to Strom et al. (31), after a median follow-up of 32 months, HDR brachytherapy (27-28 Gy in two fractions) and IMRT (74-81 Gy in 37-45 fractions) patients had significantly less deterioration in their urinary health related quality of life (HRQOL) than LDR (145 Gy in one fraction) brachytherapy patients at 1 and 3 months after irradiation. The only significant decrease in bowel HRQoL between the groups was observed 18 months after treatment, at which point IMRT patients had a slight, but significant, deterioration in their bowel HRQoL compared with HDR and LDR brachytherapy patients (31). As with brachytherapy, a heterogenous dose was prescribed to spare the urethra. The homogeneous dose distribution may affect patients in a different way than a heterogeneous brachytherapy treatment plan.

In a single-arm, phase II trial from multiple centers in Singapore (32), QoL of 74 men was assessed by EPIC at pretreatment, 12, and 24 months after SBRT of $36.25 \mathrm{~Gy}$ in 5 fractions. Overall, no significant change was observed in QoL scores over time across the three domains of urinary, bowel, and sexual scores (32). The Dutch HYPO-RT-PC trial with the updated long-term QoL analyses at baseline, the end of radiotherapy, months $3,6,12$, and 24 after radiotherapy, every other year thereafter up to 10 years, and at 
15 years using the validated Prostate Cancer Symptom Scale (PCSS) and European Organization for Research and Treatment of Cancer Quality-of-Life Questionnaire (EORTC QLQ-C30) confirmed that ultrahypofractionated radiotherapy of $42.7 \mathrm{~Gy}$ in 7 fractions $(n=583)$ was equally well tolerated as conventionally fractionated RT of $78 \mathrm{~Gy}$ in 39 fractions $(n=582)$ up to 6 years after treatment (33). These results were also consistent with the data after one year in the present study.

According to Georgetown University's report (6), which is the only existing study looking at QoL one month after SBRT of $35 \mathrm{~Gy}$ or $36.25 \mathrm{~Gy}$ in 5 fractions, the median baseline American Urological Association symptom score of 8 in 96 men significantly increased to 11 at 1 month $(p=0.001)$, but returned to baseline at 3 months $(p=0.60)$. Although a sole questionnaire after one month might have been really too short, it was conducted even in this survey by according to Georgetown University's report (6). In a meta-analysis of curative-intent SBRT for localized PC (34), the EPIC-26 was used to assess 3,293 patients. Information for urinary, bowel, and sexual domain scales was readily extractable, and the data were pooled. The EPIC urinary and bowel scores returned to baseline by 2 years post-treatment $(p=$ 0.90 and 0.09 , respectively) and did not significantly differ at 5 years post-SBRT ( $p=0.50$ and 0.80 , respectively), although these studies did not include early 1 or 2 month QoL measures. These results do not differ substantially from those of our study.

\section{Conclusions}

QoL declined at 1 month after the end of prostate SBRT, then recovered, and returned to the same level as before treatment 3-4 months after SBRT. The decrease in QoL in the first month was particularly remarkable in patients who received long-term hormone injections. One month after the end of SBRT, about $22 \%$ of people were "quite a bit or very much" troubled by the side effects of the treatment.

\section{Abbreviations}

PC

Prostate cancer

SBRT

stereotactic body radiotherapy

NCCN

National Comprehensive Cancer Network

QoL

quality of life

GU

genitourinary

GI

gastrointestinal

FACT-P

Functional Assessment of Cancer Therapy-Prostate 
WHO

World Health Organization

HIFU

high-intensity focused ultrasound

PSA

prostate-specific antigen

CTV

clinical target volume

SV

seminal vesicle

PTV

planning target volume

VMAT

volumetric modulated arc therapy

СBCT

cone-beam computed tomography

MRI

magnetic resonance imaging

CT

computed tomography

FDG

fluorodeoxyglucose

PET

positron emission tomography

PSMA

prostate specific membrane antigen

EWB

emotional well-being

FWB

family well-being

PWB

physical well-being

SWB

social well-being

PCS

prostate cancer subscale

FACT-G

FACT-General

TOI

Trial Outcome Index 
PROMS

patient-reported outcome measures

IMRT

intensity-modulated radiation therapy

LDR

low dose rate

HDR

high dose rate

EPIC

Expanded Prostate Cancer Index Composite

HRQoL

health related quality of life

PCSS

Prostate Cancer Symptom Scale

EORTC QLQ

European Organization for Research and Treatment of Cancer Quality-of-Life Questionnaire.

\section{Declarations}

Ethics approval and consent to participate: This was a retrospective study conducted with the approval of the ethics committee of the University of Tokyo (No. 3372-6). Informed consent was obtained by receiving patients' answer to the questionnaire.

Consent for publication: Not applicable.

Availability of data and materials: The datasets used and/or analyzed during the current study are available from the corresponding author on reasonable request.

Competing interests: The authors declare that they have no competing interests.

Funding: No funding was obtained for this study.

Authors' contributions: HY wrote the main manuscript text. MO, SS, YN, and OA helped to collect the data. All authors reviewed the manuscript.

Acknowledgements: Not Applicable.

\section{References}

1. Schröder FH, Hugosson J, Roobol MJ, Tammela TLJ, Ciatto S, Nelen V, et al. Screening and prostatecancer mortality in a randomized European study. N Engl J Med. 2009; 360: 1320-1328. 
2. Morgan SC, Hoffman K, Loblaw DA, et al. Hypofractionated Radiation Therapy for Localized Prostate Cancer: Executive Summary of an ASTRO, ASCO and AUA Evidence-Based Guideline. J Urol. 2019 Mar, 201(3): 528-534.

3. https://www.nccn.org/professionals/physician_gls/pdf/prostate.pdf

4. Ogita M, Yamashita H, Sawayanagi S, Takahashi W, Nakagawa K. Efficacy of a hydrogel spacer in three-dimensional conformal radiation therapy for prostate cancer. Jpn J Clin Oncol. 2020; 50: 303309.

5. https://www.facit.org/measures/FACT-P

6. Chen LN, Suy S, Uhm S, Oermann EK, Ju AW, Chen V, et al. Stereotactic body radiation therapy (SBRT) for clinically localized prostate cancer: the Georgetown University experience. Radiat Oncol. 2013; 8: 58.

7. McKay R, Haider B, Duh MS, et al. Impact of symptomatic skeletal events on health-care resource utilization and quality of life among patients with castration-resistant prostate cancer and bone metastases. Prostate Cancer Prostatic Dis. 2017 Sep; 20(3): 276-282.

8. Saad F, Ivanescu C, Phung D, et al. Skeletal-related events significantly impact health-related quality of life in metastatic castration-resistant prostate cancer: data from PREVAIL and AFFIRM trials. Prostate Cancer Prostatic Dis. 2017 Mar, 20(1): 110-116.

9. Smith MR, Shore N, Tammela TL, et al. Darolutamide and health-related quality of life in patients with non-metastatic castration-resistant prostate cancer: An analysis of the phase III ARAMIS trial. Eur J Cancer. 2021; 154: 138-146. Joseph KJ, Alvi R, Skarsgard D, et al. Analysis of health related quality of life (HRQoL) of patients with clinically localized prostate cancer, one year after treatment with external beam radiotherapy (EBRT) alone versus EBRT and high dose rate brachytherapy (HDRBT). Radiat Oncol. 2008 Jul 15; 3: 20.

10. Yokomizo A, Koga H, Ito K, et al. National Research Project on Endocrine-Radiation Combination Therapy for Locally Advanced Prostate Cancer investigators. Patient-reported outcomes following neoadjuvant endocrine therapy, external beam radiation, and adjuvant continuous/intermittent endocrine therapy for locally advanced prostate cancer: A randomized phase III trial. Cancer Med. 2021; 10(10): 3240-3248.

11. Thiery-Vuillemin A, Fizazi K, Sartor O, et al. Post Hoc Health-Related Quality of Life Analysis According to Response Among Patients with Prostate Cancer in the PROSELICA and FIRSTANA Studies. 2021; 26(7): e1179-e1188.

12. Hwang ME, Mayeda M, Liz M, et al. Stereotactic body radiotherapy with periprostatic hydrogel spacer for localized prostate cancer: toxicity profile and early oncologic outcomes. Radiat Oncol. 2019 Aug 2; 14(1): 136.

13. Stenzl A, Dunshee C, De Giorgi U, et al. Effect of Enzalutamide plus Androgen Deprivation Therapy on Health-related Quality of Life in Patients with Metastatic Hormone-sensitive Prostate Cancer: An Analysis of the ARCHES Randomised, Placebo-controlled, Phase 3 Study. Eur Urol. 2020; 78(4): 603614. Brand DH, Tree AC, Ostler P, et al. Intensity-modulated fractionated radiotherapy versus 
stereotactic body radiotherapy for prostate cancer (PACE-B): acute toxicity findings from an international, randomised, open-label, phase 3, non-inferiority trial. Lancet Oncol 2019 Nov; 20(11): 1531-1543.

14. Agarwal N, McQuarrie K, Bjartell A, et al.; TITAN investigators. Health-related quality of life after apalutamide treatment in patients with metastatic castration-sensitive prostate cancer (TITAN): a randomised, placebo-controlled, phase 3 study. Lancet Oncol. 2019; 20(11): 1518-1530. Widmark A, Gunnlaugsson A, Beckman L, et al. Ultra-hypofractionated versus conventionally fractionated radiotherapy for prostate cancer: 5-year outcomes of the HYPO-RT-PC randomised, non-inferiority, phase 3 trial. Lancet 2019; 394(10196): 385-395.

15. Tombal B, Saad F, Penson D, et al. Patient-reported outcomes following enzalutamide or placebo in men with non-metastatic, castration-resistant prostate cancer (PROSPER): a multicentre, randomised, double-blind, phase 3 trial. Lancet Oncol. 2019; 20(4): 556-569.

16. Saad F, Cella D, Basch E, et al. Effect of apalutamide on health-related quality of life in patients with non-metastatic castration-resistant prostate cancer: an analysis of the SPARTAN randomised, placebo-controlled, phase 3 trial. Lancet Oncol. 2018; 19(10): 1404-1416.

17. Unger JM, Griffin K, Donaldson GW, et al. Patient-reported outcomes for patients with metastatic castration-resistant prostate cancer receiving docetaxel and Atrasentan versus docetaxel and placebo in a randomized phase III clinical trial (SWOG S0421). J Patient Rep Outcomes. 2018; 2: 27.

18. Chi KN, Protheroe A, Rodríguez-Antolín A, et al. Patient-reported outcomes following abiraterone acetate plus prednisone added to androgen deprivation therapy in patients with newly diagnosed metastatic castration-naive prostate cancer (LATITUDE): an international, randomised phase 3 trial. Lancet Oncol. 2018; 19(2): 194-206.

19. Madsen BL, Hsi RA, Pham HT, et al. Stereotactic hypofractionated accurate radiotherapy of the prostate (SHARP), 33.5 Gy in five fractions for localized disease: First clinical trial results. Int $\mathbf{J}$ Radiat Oncol Biol Phys 2007; 67: 1099-1105.

20. Fuller DB, Falchook AD, Crabtree T, et al. Phase 2 multicenter trial of heterogeneous-dosing stereotactic body radiotherapy for low- and intermediate-risk prostate cancer: 5-year outcomes. Eur Urol Oncol 2018; 1: 540-547.

21. Meier RM, Bloch DA, Cotrutz C, et al. Multicenter Trial of Stereotactic Body Radiation Therapy for Low- and Intermediate-Risk Prostate Cancer: Survival and Toxicity Endpoints. Int J Radiat Oncol Biol Phys 2018; 102: 296-303.

22. Zelefsky M, Kollmeier M, McBride S, et al. 5-Year outcomes of a phase 1 dose escalation study using stereotactic body radiosurgery for patients with clinically localized prostate cancer. Int J Radiat Oncol 2017; 99: S156-

23. Alayed Y, Cheung P, Chu W, et al. Two StereoTactic ablative radiotherapy treatments for localized prostate cancer (2STAR): Results from a prospective clinical trial. Radiother Oncol 2019; 135: 86-90.

24. McBride SM, Wong DS, Dombrowski JJ, et al. Hypofractionated stereotactic body radiotherapy in low-risk prostate adenocarcinoma: Preliminary results of a multi-institutional phase 1 feasibility trial. 
Cancer 2012; 118: 3681-3690.

25. Loblaw A, Cheung P, D'Alimonte $L$, et al. Prostate stereotactic ablative body radiotherapy using a standard linear accelerator: Toxicity, biochemical, and pathological outcomes. Radiother Oncol 2013; 107: 153-158.

26. Hannan R, Tumati V, Xie X-J, et al. Stereotactic body radiation therapy for low and intermediate risk prostate cancer-Results from a multi-institutional clinical trial. Eur J Cancer 2016; 59: 142-151.

27. Kretschmer A, Ploussard G, Heidegger I, et al. Health-related Quality of Life in Patients with Advanced Prostate Cancer: A Systematic Review. Eur Urol Focus. 2020 Feb 20: S2405-4569(20)30064-X.

28. Feigenberg SJ, Lee WR, Desilvio ML, et al. Health-related quality of life in men receiving prostate brachytherapy on RTOG 98-05. Int J Radiat Oncol Biol Phys. 2005 Jul 15; 62(4): 956-964. Wei JT, Dunn RL, Litwin MS, et al. Development and validation of the expanded prostate cancer index composite (EPIC) for comprehensive assessment of health-related quality of life in men with prostate cancer. Urology 2000; 56: 899-905.

29. Thiery-Vuillemin A, Fizazi K, Sartor O, et al. An analysis of health-related quality of life in the phase III PROSELICA and FIRSTANA studies assessing cabazitaxel in patients with metastatic castrationresistant prostate cancer. ESMO Open. 2021; 6(2): 100089. Wilkins A, Mossop H, Syndikus I, et al. Hypofractionated radiotherapy versus conventionally fractionated radiotherapy for patients with intermediate-risk localised prostate cancer: 2-year patient-reported outcomes of the randomised, noninferiority, phase 3 CHHiP trial. Lancet Oncol 2015 Dec; 16(16): 1605-1616. Tan JSH, Teh JYH, Tan LLY, et al. Efficacy, toxicity, and quality-of-life outcomes of ultrahypofractionated radiotherapy in patients with localized prostate cancer: A single-arm phase 2 trial from Asia. Asia Pac J Clin Oncol 2021; Dec 14. doi: 10.1111/ajco.13742. Online ahead of print.

30. Slevin F, Sethugavalar B, Al-Qaisieh B, et al. Ten-year longitudinal health-related quality of life following iodine-125 brachytherapy monotherapy for localized prostate cancer. J Contemp Brachytherapy. 2020 Dec; 12(6): 540-546.

31. Strom TJ, Cruz AA, Figura NB, et al. Health-related quality-of-life changes due to high-dose-rate brachytherapy, low-dose-rate brachytherapy, or intensity-modulated radiation therapy for prostate cancer. Nov-Dec 2015; 14(6): 818-825.

32. Tan JSH, Teh JYH, Tan LLY, et al. Efficacy, toxicity, and quality-of-life outcomes of ultrahypofractionated radiotherapy in patients with localized prostate cancer: A single-arm phase 2 trial from Asia. Asia Pac J Clin Oncol 2021; Dec 14. doi: 10.1111/ajco.13742. Online ahead of print.

33. Fransson $P$, Nilsson $P$, Gunnlaugsson $A$, et al. Ultra-hypofractionated versus conventionally fractionated radiotherapy for prostate cancer (HYPO-RT-PC): patient-reported quality-of-life outcomes of a randomised, controlled, non-inferiority, phase 3 trial. Lancet Oncol 2021; 22(2): 235-245.

34. Jackson WC, Silva J, Hartman HE, et al. Stereotactic body radiation therapy for localized prostate cancer: a systematic review and meta-analysis of over 6,000 patients treated on prospective studies. Int J Radiat Oncol Biol Phys. 2019; 104(4): 778-789. 


\section{Figures}

\section{Figure 1}

Mean Functional Assessment of Cancer Therapy-Prostate (FACT-P) Trial Outcome Index score (TOI, blue line), Functional Assessment of Cancer Therapy-General (FACT-G) Total score (orange line), and FACT-PTotal score (gray line). Time from last SBRT dose (months).

\section{Figure 2}

Mean Functional Assessment of Cancer Therapy-Prostate (FACT-P) Trial Outcome Index (TOI) score (2-a) and mean FACT-P-Total score (2-b) by no (blue line), short term (orange line), and long term (gray line) hormonal therapy.

\section{Figure 3}

Mean four subscale scores. P6 (I have trouble moving my bowels): blue, P7 (I have difficulty urinating): orange, BL2 (I urinate more frequently than usual): gray, and P8 (My problems with urinating limit my activities): yellow line.

\section{Figure 4}

The distribution of answers to subscale GP5 ( am bothered by side effects of treatment (not at all: dark blue, a little bit: orange, somewhat: gray, quite a bit: yellow, and very much: sky blue). 